\title{
Shock waves promote spinal cord repair via TLR3
}

\author{
Can Gollmann-Tepeköylü,' Felix Nägele, ${ }^{1}$ Michael Graber, ${ }^{1,2}$ Leo Pölzl, ${ }^{1,2}$ Daniela Lobenwein,, ${ }^{1,2}$ \\ Jakob Hirsch, ${ }^{1}$ Angela An, ${ }^{1}$ Regina Irschick, ${ }^{2}$ Bernhard Röhrs, ${ }^{3}$ Christian Kremser, ${ }^{4}$ Hubert Hackl, ${ }^{5}$ \\ Rosalie Huber, ${ }^{1}$ Serena Venezia,, ${ }^{6}$ David Hercher, ${ }^{7,8}$ Helga Fritsch, ${ }^{2}$ Nikolaos Bonaros, ${ }^{1}$ \\ Nadia Stefanova, ${ }^{6}$ Ivan Tancevski, ${ }^{9}$ Dirk Meyer, ${ }^{3}$ Michael Grimm, ${ }^{1}$ and Johannes Holfeld ${ }^{1,8}$ \\ 'Department of Cardiac Surgery and 'Division of Clinical and Functional Anatomy, Medical University of Innsbruck, \\ Innsbruck, Austria. Institute of Molecular Biology/Center for Molecular Biosciences Innsbruck, University of Innsbruck, \\ Innsbruck, Austria. ${ }^{4}$ Department of Radiology, ${ }^{5}$ Division of Bioinformatics, Biocenter, and ${ }^{6}$ Division of Neurobiology, \\ Department of Neurology, Medical University of Innsbruck, Innsbruck, Austria. ${ }^{7}$ Ludwig Boltzmann Institute for \\ Experimental and Clinical Traumatology, Vienna, Austria. ${ }^{8}$ Austrian Cluster for Tissue Regeneration, Vienna, Austria. \\ ${ }^{9}$ Department of Internal Medicine II, Infectious Diseases, Pneumology, Rheumatology, Medical University of Innsbruck, \\ Innsbruck, Austria.
}

\begin{abstract}
Spinal cord injury (SCI) remains a devastating condition with poor prognosis and very limited treatment options. Affected patients are severely restricted in their daily activities. Shock wave therapy (SWT) has shown potent regenerative properties in bone fractures, wounds, and ischemic myocardium via activation of the innate immune receptor TLR3. Here, we report on the efficacy of SWT for regeneration of SCI. SWT improved motor function and decreased lesion size in WT but not $\mathrm{Tlr}^{\mathrm{-1}}$ mice via inhibition of neuronal degeneration and IL6-dependent recruitment and differentiation of neuronal progenitor cells. Both SWT and TLR3 stimulation enhanced neuronal sprouting and improved neuronal survival, even in human spinal cord cultures. We identified $t / r 3$ as crucial enhancer of spinal cord regeneration in zebrafish. Our findings indicate that TLR3 signaling is involved in neuroprotection and spinal cord repair and suggest that TLR3 stimulation via SWT could become a potent regenerative treatment option.
\end{abstract}

Authorship note: CGT and FN contributed equally to this work.

Conflict of interest: $\mathrm{JH}$ and MC are shareholders of Heart Regeneration Technologies, a Medical University of Innsbruck spin-off that aims to promote cardiac shock wave therapy.

Copyright: () 2020, American Society for Clinical Investigation.

Submitted: October 25, 2019

Accepted: June 24, 2020

Published: August 6, 2020.

Reference information: JCI Insight. 2020;5(15):e134552. https://doi.org/10.1172/jci. insight.134552.

\section{Introduction}

In a recent study, we showed improvement of locomotor function, a reduction of degenerating neurons, and improved survival upon shock wave therapy (SWT) in a model of spinal cord ischemia (1). However, spinal cord ischemia with sudden disruption of spinal blood supply accounts for only a very small fraction of spinal cord injuries (SCIs). In contrast, traumatic SCI represents a severe socioeconomic health burden, accounting for $>90 \%$ of all SCIs $(2,3)$. Traumatic SCI after severe impaction causes serious damage of the spinal cord with (a) contusion injury and necrotic or apoptotic death of neuronal cells; (b) release of cytokines and toxins from injured glial cells, causing secondary damage in neighboring cells and eliciting an inflammatory response, leading to (c) activation of resident astrocytes and pericytes and recruitment of peripheral immune cells and fibroblasts; and, (d) eventually, the formation of glial and fibrotic scars at the damage site (4). TLRs are a part of the innate immune system that mainly bind highly conserved sequences of microorganisms. However, as ligand selectivity is relatively low, TLRs can recognize endogenous molecules released during inflammation and tissue damage (5). In particular, TLR3 has recently been characterized as a receptor activated by endogenous danger signals - mainly RNA - released upon tissue damage or cellular stress $(5,6)$. Sensing of tissue damage and subsequent orchestration of the inflammatory response via TLR3 might thus represent an innate mechanism of tissue regeneration (7).

SWT has been described to induce tissue regeneration through stimulation of the innate immune receptor TLR3 in skin, bone, tendons, and cardiac tissue (8-10). Here, we aimed to substantiate (a) the physiological role of TLR3 in spinal cord repair after injury and (b) the efficacy of TLR3 stimulation via SWT in traumatic SCI. 


\section{Results}

Improved locomotor function and decreased lesion size after treatment. To investigate the effect of SWT on spinal regeneration, we used a model of chronic spinal cord contusion injury in WT and Tlr $3^{-/}$mice and applied SWT 2 weeks after injury (Figure 1A). SWT markedly improved locomotor function in WT animals in comparison with untreated control animals, whereas SWT had no effect in Tlr3-deficient mice (Figure 1B). Analogously, SWT markedly extended the distance covered in WT but not in Tlr3 ${ }^{-1-}$ animals, as measured via automated open-field analysis (Figure 1C).

Previously, our group showed that SWT reduces scarring after myocardial infarction (10). To analyze whether SWT may decrease lesion size after SCI, we performed MRI of the spinal cord (Figure 1D). MRI revealed a significant reduction of lesion size 10 weeks after injury in SWT-treated WT mice compared with that in untreated controls (Figure 1E). SWT had no regenerative effect on the spinal cords in Tlr3-deficient animals. A finely tuned interplay between parasympathetic efferents from the sacral micturition center, providing excitatory signals for detrusor contraction, and somatic efferents from the pudendal nerve, inhibiting the external urethral sphincter, guarantees micturition. However, after SCI detrusor sphincter dyssynergia can cause neurogenic bladder dysfunction (11). As an incidental finding, we observed increased bladder volumes, reflecting a detrusor sphincter dyssynergia in control and Tlr $3^{-1-}$ animals, whereas SWT-treated animals had reduced bladder volumes (Figure 1F). In line with MRI findings, spinal cord sections revealed decreased lesion size in SWT-treated mice (Figure 2, A and B). Secondary damage upon SCI is characterized by a biochemical cascade resulting in neuronal degeneration and further loss of function (12). SWT reduced the number of degenerating neurons 72 hours and 10 weeks after treatment (Figure $2 \mathrm{C}$ ). No beneficial effect of SWT on the number of degenerating neurons was observed in $T l r 3^{-1-}$ mice (Figure 2, D and E).

TLR3 is neuroprotective in a model of SCI in zebrafish. Zebrafish are characterized by a remarkable regenerative capacity, with robust axonal growth and, as a consequence, extensive restoration of locomotor function; therefore, they represent a well-established model to investigate spinal cord regeneration (1315). As regeneration involves signaling processes conserved between mammals and fish (16), we aimed to further substantiate the importance of TLR3 signaling for spinal cord protection in zebrafish. After a lesion was created on the spinal cord, larvae were left untreated (controls), exposed to an inhibitor of TLR3 signaling, or exposed to TLR3 agonist poly(I:C) (Figure 3A). We found evidence for enhanced neuroprotection after TLR3 stimulation: poly(I:C)-treated fish exhibited increased neuron volume within the lesion area, whereas inhibition of TLR3 signaling resulted in decreased neuroprotection (Figure $3, \mathrm{~B}$ and C). In accordance, TLR3 stimulation improved locomotor activity after spinal cord lesion, whereas TLR3 inhibition resulted in impaired locomotion (Figure 3, D and E). These findings suggest TLR3-mediated neuroprotection after SCI in zebrafish

SWT regulates IL-6 expression and promotes differentiation of neuronal progenitor cells. To substantiate the underlying mechanisms of SWT for spinal cord repair, we treated cultured human neurons with SWT and performed RNA-Seq analysis thereafter. Genes with most significant regulation are depicted in Figure 4A: importantly, RNA-Seq analysis revealed induction of both IL-6 signaling as well as of neuronal differentiation. Thus, we hypothesized that SWT might promote recruitment and differentiation of neuronal progenitor cells (NPCs), possibly via IL-6. NPCs are a crucial source for newly forming neurons after injury, and IL-6 is pivotal for both postnatal recruitment and differentiation of NPCs (17). Moreover, IL-6 is upregulated upon TLR3 activation via TIR domain-containing adapter-inducing IFN- $\beta$ (TRIF) and nuclear translocation of NF- $\kappa \mathrm{B}(18)$ (Supplemental Figure 1; supplemental material available online with this article; https://doi.org/10.1172/jci. insight.134552DS1). Indeed, SWT resulted in increased TLR3 expression (Figure 4B), with associated upregulation of TRIF (Figure 4C), IL-6 (Figure 4D), and IL-6 receptor (Figure 4E). To prove recruitment of NPCs in vivo, next we quantified cells positive for the neuroectodermal stem cell marker nestin in spinal cord sections after SWT (Figure 5A). We detected a significant increase of nestin-positive cells in SWT-treated animals, which by far exceeded those in sham-treated animals or controls (Figure 5B), indicative of either increased recruitment or possibly enhanced local proliferation of NPCs. To further substantiate a putative direct effect of SWT on progenitors, we isolated NPCs from spinal cords obtained from WT or Tlr3 ${ }^{-1}$ mice for further in vitro testings. Isolated NPCs were positive both for nestin and Tlr3 (Figure 5, C and D). SWT as well as poly(I:C) treatment did not affect proliferation in both NPCs derived from WT and from $T l r 3^{-/-}$mice (Figure 5E). However, both SWT and poly(I:C) exposure significantly enhanced NPC differentiation, profoundly increasing the number of cells that expressed the neuronal cell marker $\beta$ III tubulin (Figure 5F). Importantly, the positive effect on neuronal differentiation of either poly(I:C) or SWT was TLR3 dependent (Figure 5G). 
A
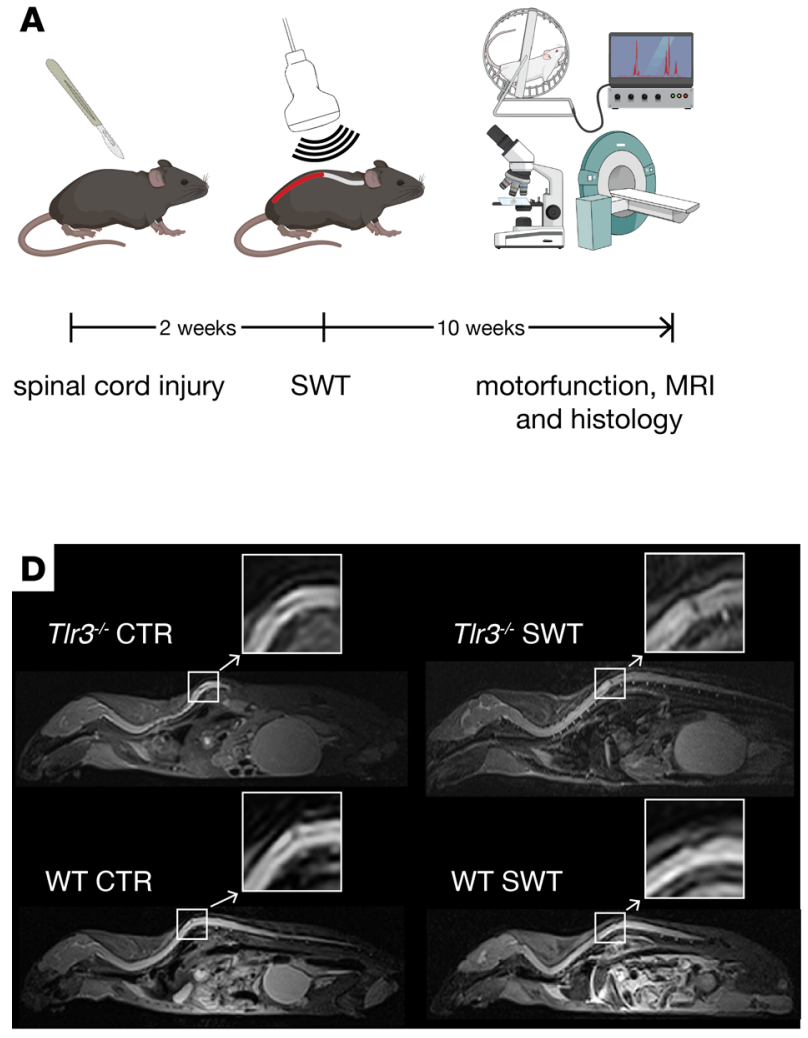

B

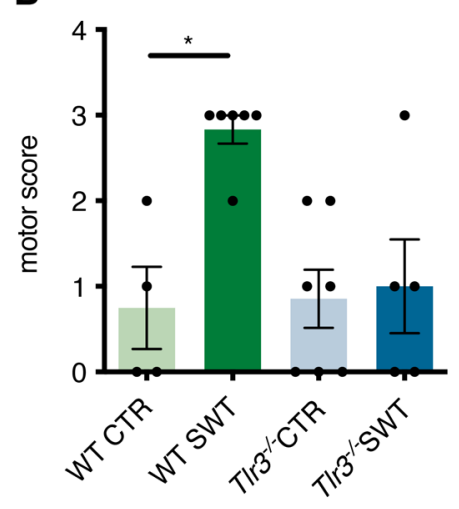

$\mathbf{E}$

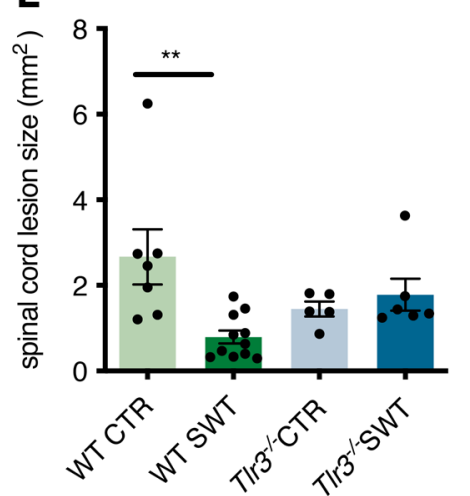

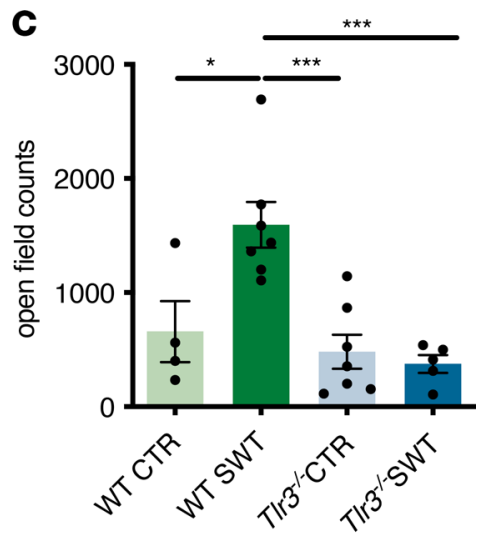

$\mathbf{F}$

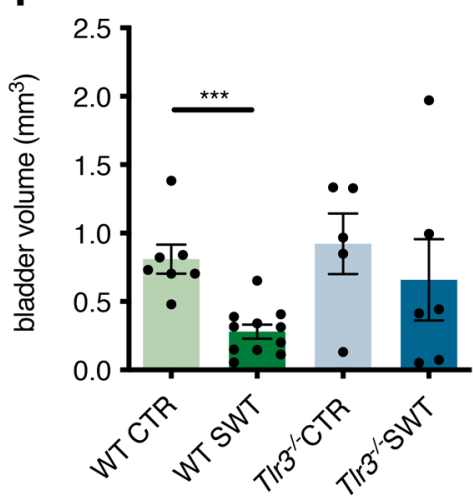

Figure 1. Improved locomotor function and decreased lesion size after treatment. (A) Mice were treated 2 weeks after spinal cord injury, and analyses were performed 10 weeks after treatment. (B) Gait analysis revealed improved motor scores in SWT-treated WT animals compared with controls, whereas SWT had no effect in TIr3-deficient mice. ${ }^{*} P<0.05 . n=4$ (WT CTR), $n=6$ (WT SWT), $n=7$ (T/r3 $3^{-1-}$ CTR), $n=5$ (TIr $3^{-1-}$ SWT). (C) Covered distance in automated open-field analysis was significantly increased by SWT of WT animals. SWT had no beneficial effect in T/r $3^{-/-}$mice. ${ }^{*} P<0.05,{ }^{* *} P<0.001 . n=4$ (WT CTR), $n=7$ (WT SWT), $n=7$ (T/r3 ${ }^{-1-}$ CTR), $n=5$ (Tlr3 ${ }^{-1-}$ SWT). (D) For quantification of lesion size, we performed MRI analysis of the spinal cord (original magnification, $\times 3.5$, insets). (E) SWT resulted in a significant reduction of spinal cord lesion size 10 weeks

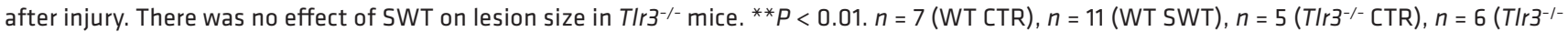
SWT). (F) SWT resulted in decreased bladder volumes in WT but not TIr $3^{-1-}$ mice. ${ }^{* * *} P<0.001 . n=7$ (WT CTR), $n=11$ (WT SWT), $n=5$ (TIr3 ${ }^{-1-}$ CTR),

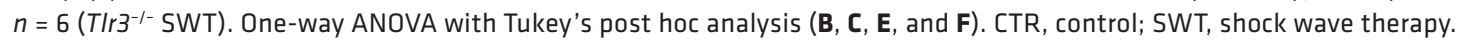

SWT enhances neuronal sprouting, reduces apoptosis, and inhibits neuronal degeneration. We finally set out to determine which effects SWT may exert on primary murine neurons, as opposed to NPCs, that could beneficially affect the reestablishment of motoneuron and ultimately locomotor function after SCI. For this purpose, we isolated dorsal root ganglia (DRG) neurons obtained from WT and Tlr3-deficient mice and exposed them to SWT or poly(I:C) treatment, respectively (Figure 6A). SWT improved viability of WT but not $\mathrm{Tlr}^{-{ }^{--}}$neurons in an XTT assay, whereas poly(I:C) showed no effect (Figure 6B). Primary damage of the spinal cord upon injury is followed by a secondary damage. Release of neurotoxic mediators triggers neuronal apoptosis and contributes to further extension of the damage. Thus, limitation of secondary damage exerts a benefit on spinal cord function after injury (19). Upon FCS starvation, SWT protected WT neurons from apoptotic cell death. Poly(I:C) treatment reduced neuronal apoptosis after starvation; however, the reduction was not significant (Figure 6C). Axonal sprouting is crucial for restoration of spinal cord function after injury (20). SWT stimulated neuronal sprouting in WT cells, whereas it had no effect in Tlr $3^{-1-}$ cells. Poly(I:C) stimulation showed no effect on neuronal sprouting neither in WT or Tlr3 ${ }^{-1-}$ cells (Figure 6, D and E).

To confirm the neuroprotective SWT effects in tissue, we performed a murine spinal slice culture (Figure 7A). Consistently, both SWT and poly(I:C) significantly reduced the number of degenerating neurons compared with untreated controls (Figure 7B). However, the protective effects were missing in cultures obtained from $\mathrm{Tlr}^{-3^{-1}}$ mice. To extend our investigation to a human model, we examined the effect of SWT in human spinal slice cultures obtained from the middle or lower cervical segments of the spinal cord (Figure 7C). SWT indeed reduced the number of degenerating neurons in human tissue. In addition, a significant, albeit less pronounced effect was also observed upon addition of the 
A

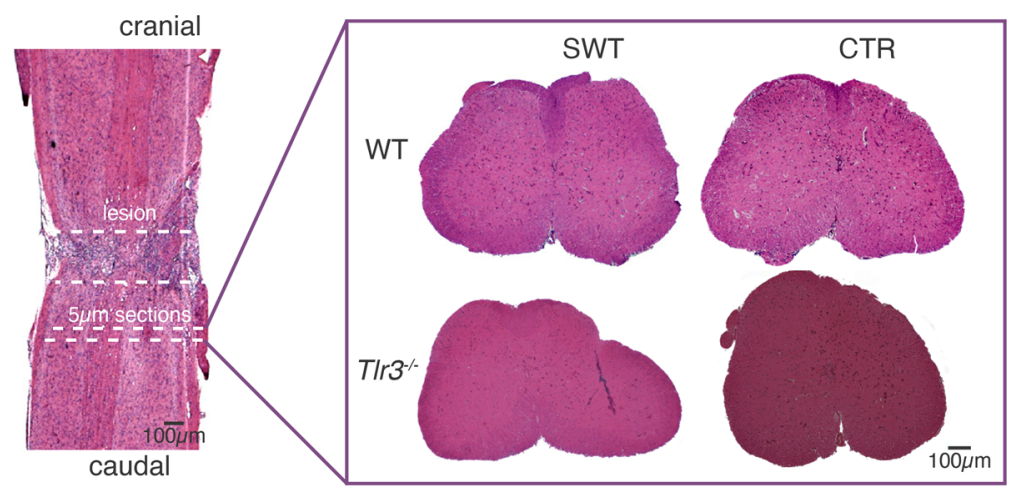

\section{C}

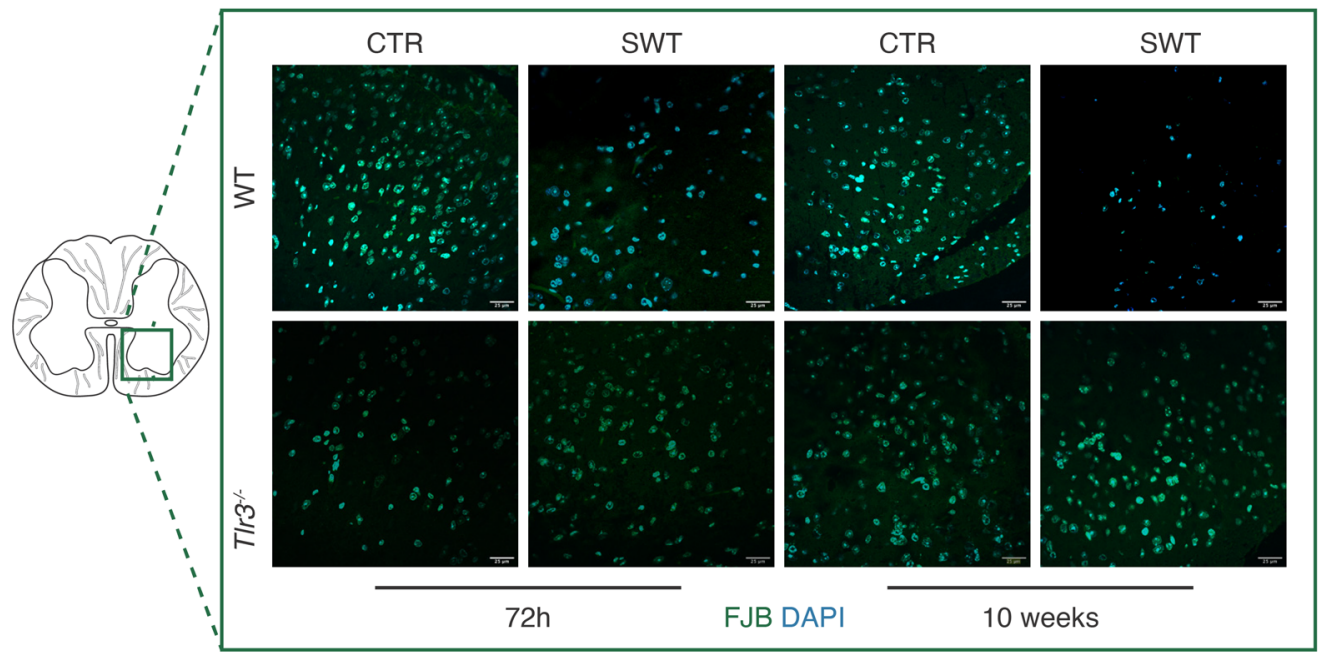

B

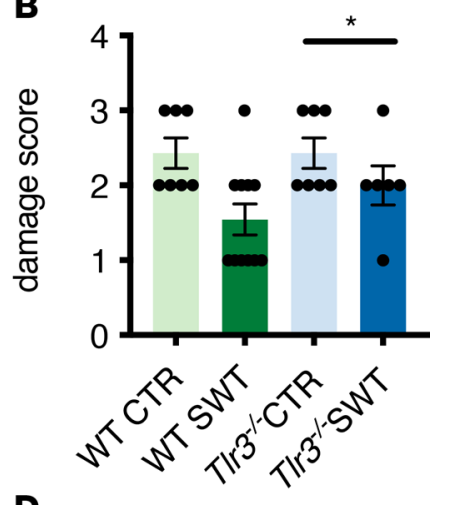

D

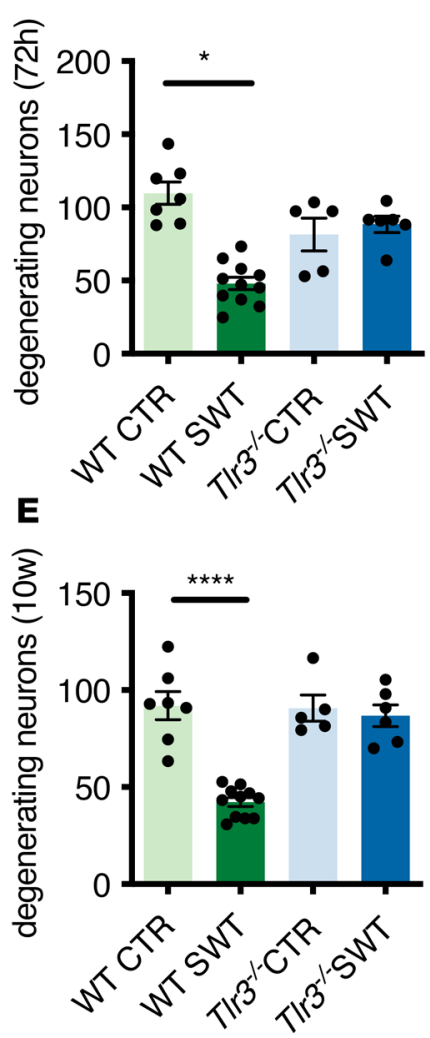

Figure 2. SWT reduces spinal cord damage and neuronal degeneration. (A and B) Histological sections of the lesion revealed decreased lesion

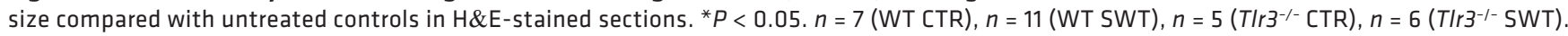
Scale bar: $100 \mu \mathrm{m}$. (C) Fluoro-Jade B staining of spinal cord sections obtained 72 hours or 10 weeks after treatment of WT or T/r3-knockout mice. Scale bar: $25 \mu \mathrm{m}$. (D) SWT exerted a neuroprotective effect resulting in decreased neuronal degeneration 72 hours after treatment in WT mice. The

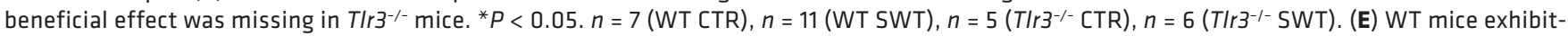
ed significantly reduced numbers of degenerating neurons after treatment compared with untreated controls. T/r3 $3^{-1-}$ animals did not benefit from

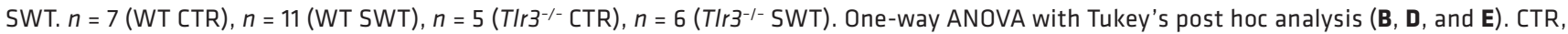
control; SWT, shock wave therapy; FJB, Fluor-Jade B.

TLR3 agonist poly(I:C) (Figure 7D). In summary, SWT markedly reduced neuronal degeneration in vitro, both in murine and in human tissue culture.

\section{Discussion}

The present study provides evidence that SWT holds significant potential as therapeutic approach for the treatment of chronic SCI and presents mechanistic insight into its mode of regeneration. SWT caused a significant improvement of locomotor function compared with untreated controls. Here, we show for the first time to our knowledge a dramatic effect on the restoration of motor function in a chronic model of SCI. The beneficial effect of SWT on locomotor function was reflected by, and presumably 
A

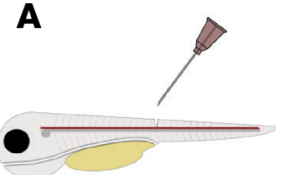

Spinal cord injury \&

Treatment with

TLR3-INH vs. Poly(I:C)
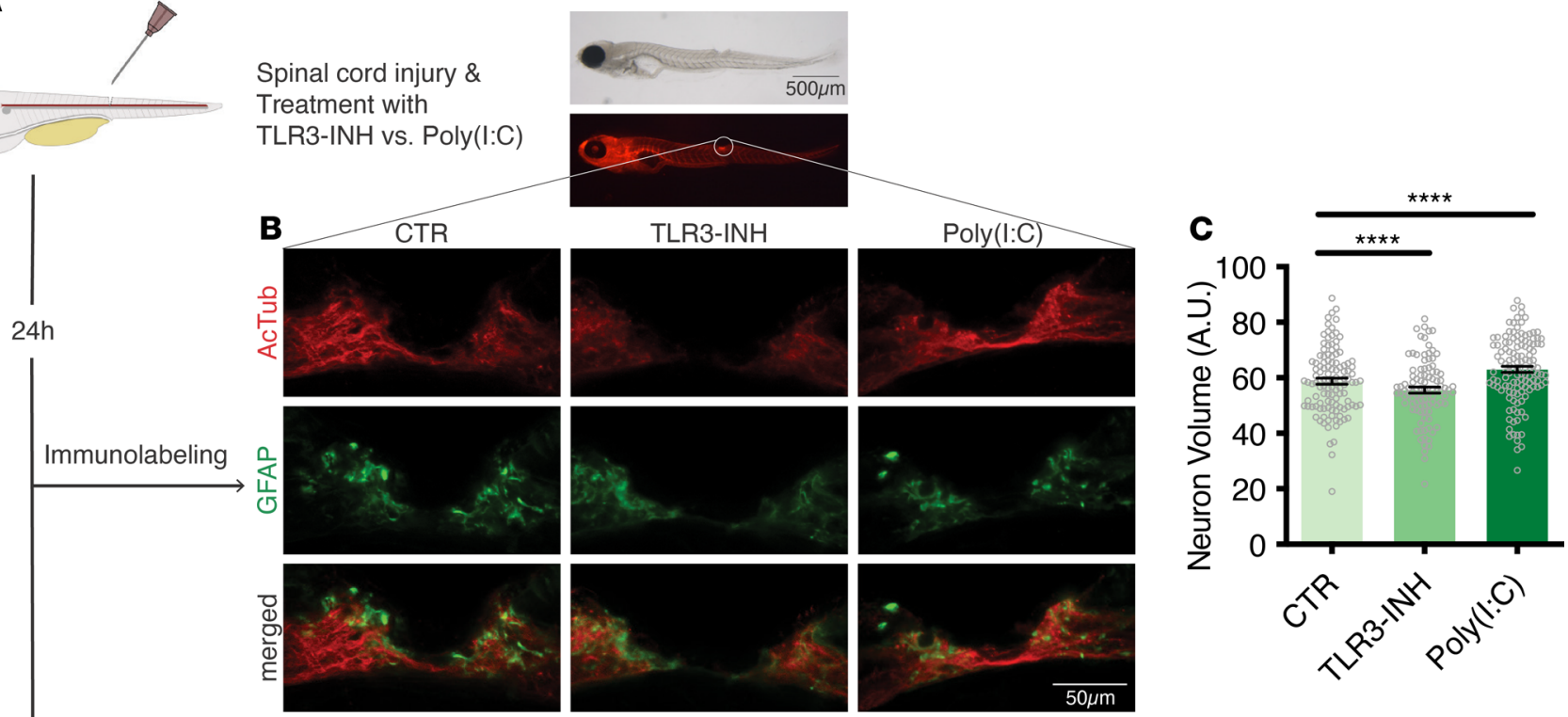

Immunolabeling
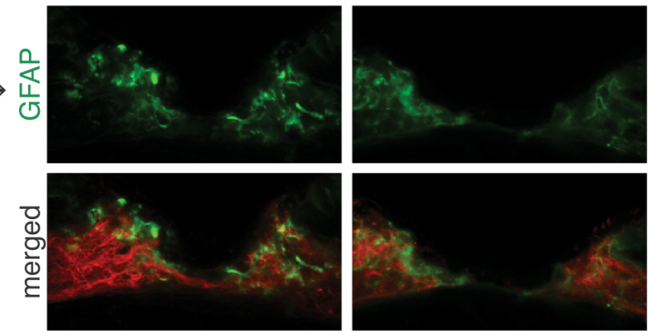

D
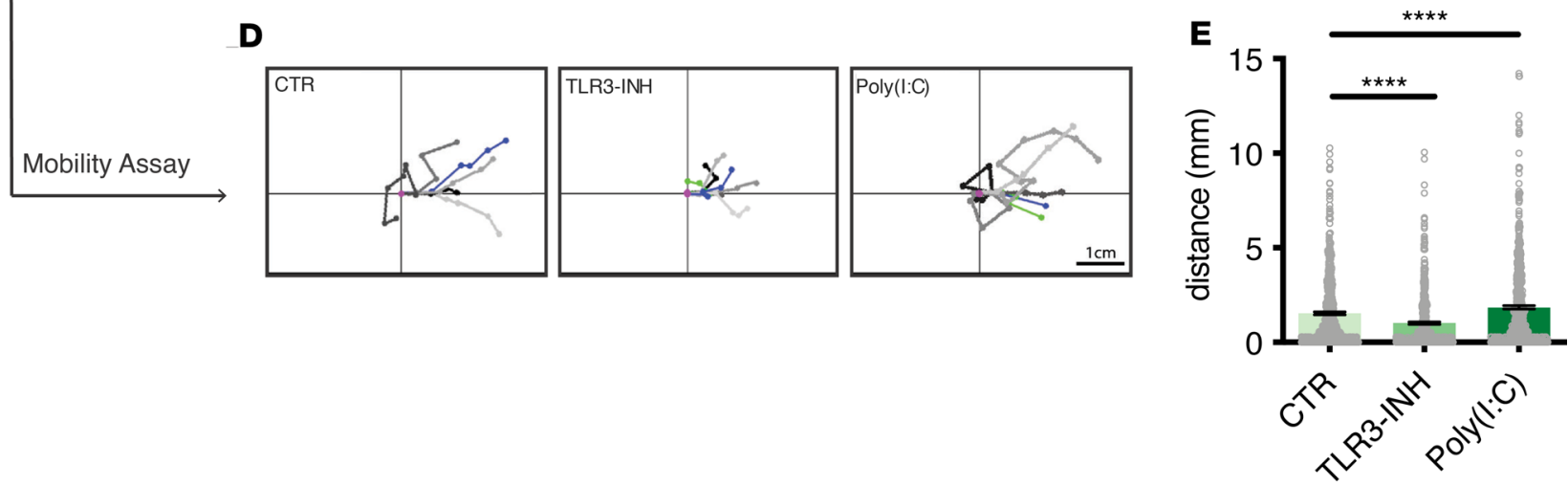

Figure 3. TLR3 is neuroprotective in a zebrafish spinal cord injury model. (A) After spinal cord injury zebrafish were treated with TLR3 agonist poly(I:C) or TLR3 inhibitor. (B) Representative images showing a neuroprotective effect of TLR3 stimulation after spinal cord injury. Scale bar: $50 \mu \mathrm{m}$ (bottom); $500 \mu \mathrm{m}$ (top). (C) We found significantly increased neuron volumes in poly(I:C)-treated fish, whereas pharmacological inhibition of TLR3 resulted in decreased neuroprotection. ${ }^{* * *} P<0.0001 . n=117$ (CTR), $n=127$ (poly(I:C)), $n=98$ (TLR3-INH). (D) Swimming distance of the zebrafish after TLR3 stimulation or inhibition was quantified. Scale bar: $1 \mathrm{~cm}$. (E) TLR3 stimulation improved locomotor activity after spinal cord lesion, and TLR3 inhibition resulted in impaired locomotion in zebrafish. ${ }^{* * *} P<0.0001 . n=64$ (CTR), $n=67$ (poly(I:C)), $n=73$ (TLR3-INH). One-way ANOVA with Tukey's post hoc analysis (C and E). CTR, control; TLR3-INH, TLR3 inhibitor.

functionally linked to, decreased spinal lesion size after treatment. Reduction of secondary damage has been proposed as a key approach for spinal cord regeneration (20). At least to some extent, this may be the result of a reduction of degenerating neurons, an effect seen as early as 72 hours after treatment and persisting for 10 weeks. Importantly, we have the first evidence to our knowledge for a neuroprotective effect in human spinal cord tissue. As an incidental finding, we observed decreased bladder volumes after SWT, indicating alleviation of detrusor sphincter dyssynergia. Underlying mechanisms for the observed phenomenon remain unclear and should be addressed in future studies.

We also report some genuine cell-intrinsic alterations, such as enhanced differentiation in cultures of mouse NPCs and enhanced neuronal sprouting, diminished apoptosis, and stimulated proliferation in cultures of mouse primary neurons. Most of these effects could not be mimicked by poly(I:C) exposure but were strictly dependent on TLR3. Both TLR3 stimulation as well as SWT have been described to induce release of growth factors, including VEGF, FGF, and NGF $(10,21)$. Consistently, we observed upregulation of VEGF and its receptor VEGFR2 (Supplemental Figure 2). We therefore presume that at least some of the regenerative effects might be due to the induction of angiogenesis.

Both in vitro as well as in vivo data strongly suggest the involvement of TLR3 signaling in spinal cord regeneration, in line with previous findings on cardiac regeneration $(22,23)$. TLR3 is expressed 
A

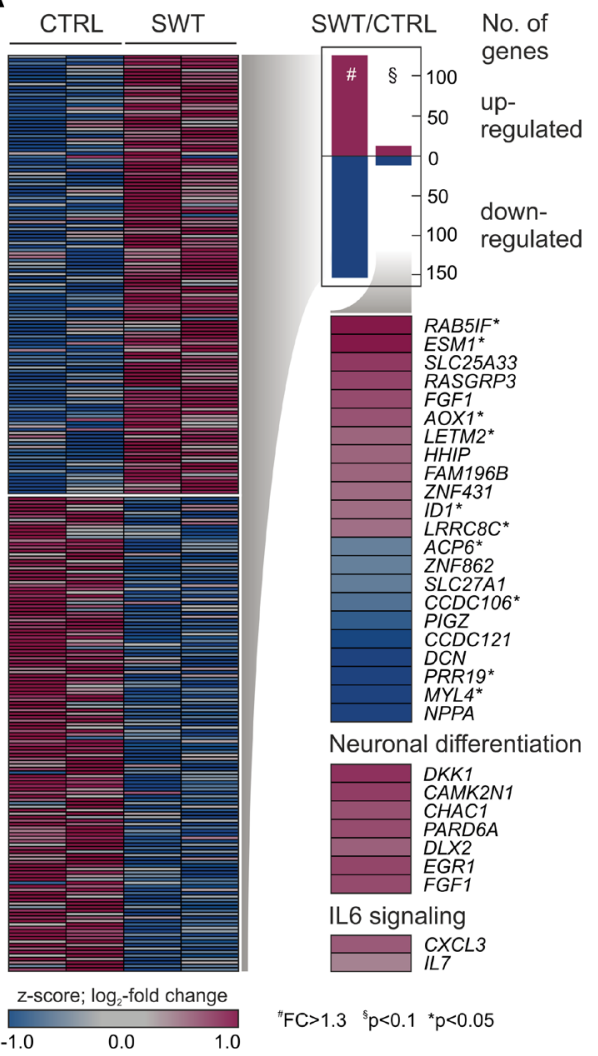

B

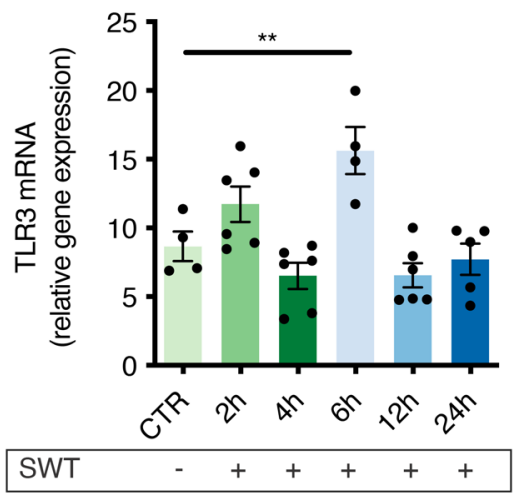

D

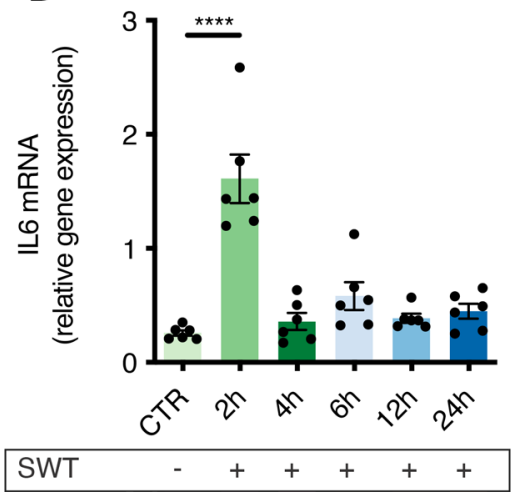

C

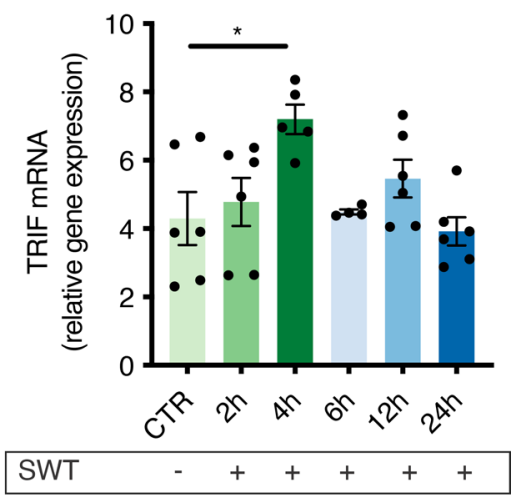

E

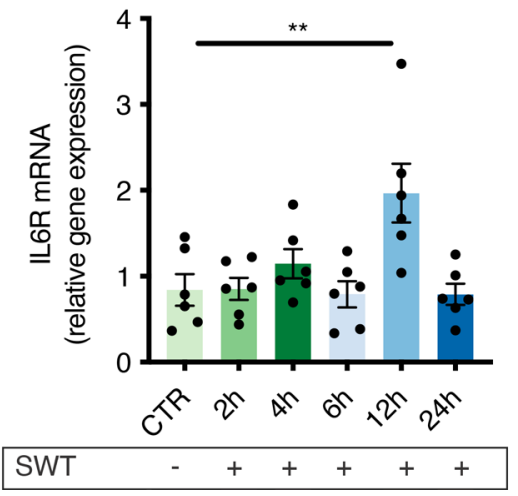

Figure 4. SWT regulates IL-6 expression. (A) RNA-Seq revealed a different gene expression profile in SH-SY5Y cells treated with SWT compared with untreated controls. (B) TLR3 regulation after SWT. ${ }^{* *} P<0.01 . n=4-6$. (C) TRIF regulation after SWT. ${ }^{*} P<0.05$. $n=4-6$. (D) IL-6 regulation after SWT. ${ }^{* * * *} P<0.0001 . n=6$. (E) IL-6 receptor regulation after SWT. ${ }^{* *} P<0.01 . n=6$. One-way ANOVA with Tukey's post hoc analysis (B-E). CTR, control; SWT, shock wave therapy; TRIF, TIR domain-containing adapter-inducing IFN- $\beta$; IL6R, IL-6 receptor.

throughout the entire spinal cord tissue, including microglia, astrocytes, oligodendrocytes, and neurons (24). TLR3 stimulation releases neuroprotective mediators on astrocytes (21). Further studies are needed to elucidate whether TLR3-mediated SWT effects are mainly attributed to one specific cell type within the spinal cord or whether they represent an interplay of different cell types.

DAMPs, including RNA, proteins, or lipids, are released upon cellular injury and can activate TLR (25). SWT causes the release of specific exosomes mediating angiogenesis and repair (26). Exosomes can activate TLRs either by activating membrane proteins or their cargo (27). Whether the released exosomes are indeed the TLR3 ligands following SWT has to be addressed in the future.

The modified inflammation after SWT was also reflected by the downregulation of TLR4 expression, the activation of which has been repeatedly associated with tissue damage following SCI (28). Human tissue slices showed significantly diminished TLR4 expression after SWT (Supplemental Figure 3). In addition, we found upregulation of IL-6 and consecutive NPC recruitment and differentiation. Thereby, we found rapid upregulation of IL- 6 with a delayed transcription of TLR3 and TRIF upon SWT. IL-6 promotes regeneration and repair in SCI (29). It is transcribed rapidly upon TLR3 activation via recruitment of TRIF and TRAF6, with subsequent nuclear translocation of NF- $\mathrm{KB}(30)$. TLR3 and TRIF are upregulated in an autocrine manner upon activation, possibly explaining the delayed regulation upon SWT in comparison with IL-6.

This study has the following limitations. As the described mouse model represents a model of chronic SCI and the zebrafish model represents an acute model of injury, results cannot be directly translated. Due to the invasive animal model, sample size was low in the animal experiments. The main purpose behind the MRI analysis was the quantification of spinal cord lesion size. While analyzing the images, we incidentally discovered differences in bladder volumes. Therefore, we did not standardize the voiding protocol before MRI measurements. 


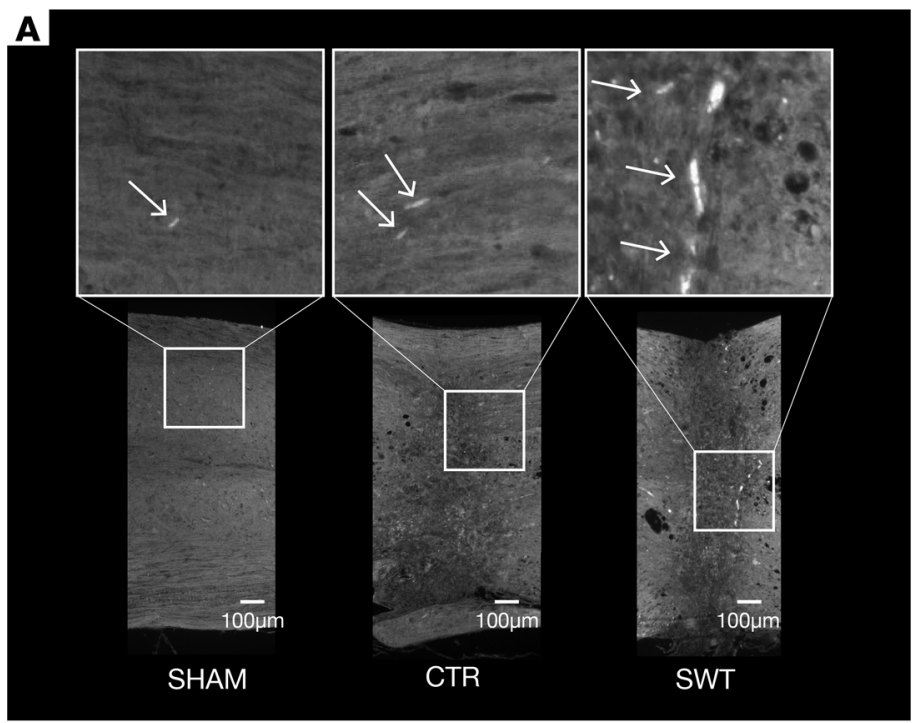

E

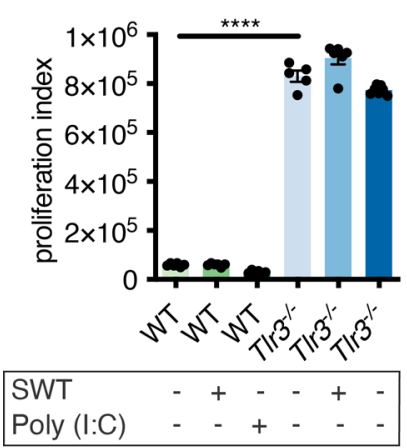

$\mathbf{F}$
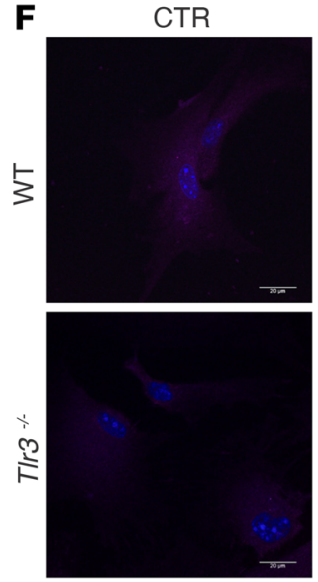

B

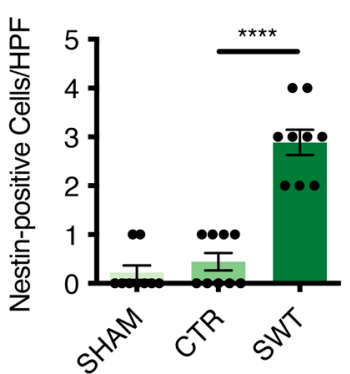

D

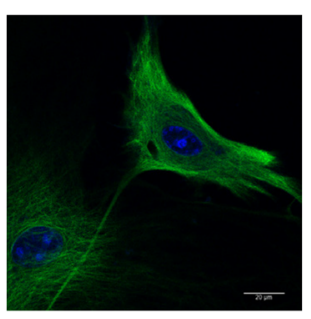

Nestin DAPI
C

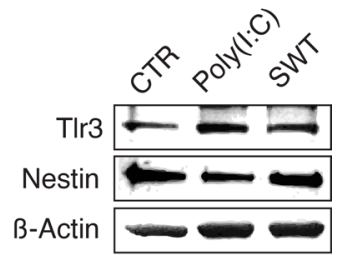

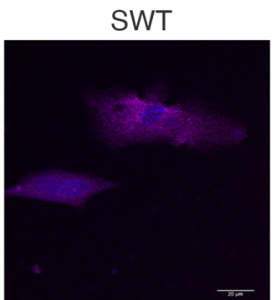

Poly(I:C)

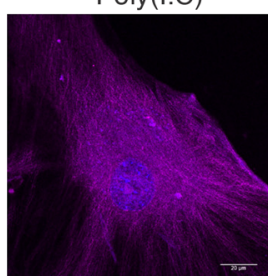

G

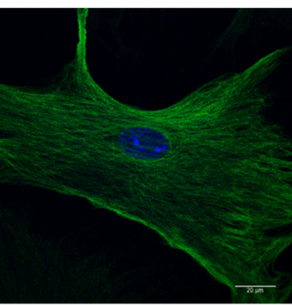

要
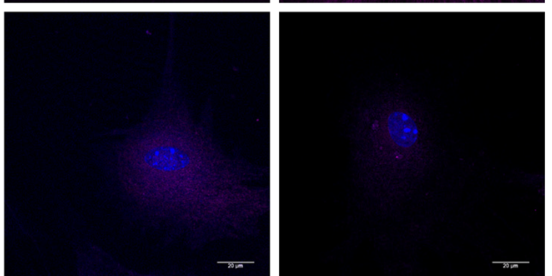

beta III - tubulin DAPI

Figure 5. SWT promotes differentiation of neuronal progenitor cells. (A) To investigate possible involvement of NPCs, we quantified nestin-positive cells in spinal cord sections obtained from SWT-treated WT mice. Scale bar: $100 \mu \mathrm{m}$. (B) We found a significant increase of nestin-positive cells after treatment, suggesting involvement of NPCs in SWT-induced spinal regeneration. ${ }^{* * *} P<0.0001 . n=9$ (Sham), $n=9$ (CTR), $n=9$ (SWT). (C and D) NPCs were isolated from WT and T/r3 ${ }^{-/-}$mice. We found abundant expression of T/r3 and nestin in NPCs. Treatment of NPCs with SWT or poly(I:C) increased T/r3 expression. Scale bar: $20 \mu \mathrm{m}$. (E) SWT and poly(I:C) treatment did not affect NPC proliferation, neither in NPCs derived from WT mice nor in those from TIr3-knockout mice. NPCs obtained from TIr3-deficient mice showed significantly increased proliferation rates compared with WT NPCs. ${ }^{* * *} P<0.0001 . n=6-7$. (F) NPCs were treated with SWT or poly(I:C), respectively, and analyzed for the expression of the neuronal cell marker $\beta$ III tubulin subsequently. Scale bar: $20 \mu \mathrm{m}$. (G) Both SWT and poly(I:C) exposure significantly enhanced NPC differentiation in WT but not $\mathrm{Tlr}^{-1-}$ cells, increasing the number of cells expressing $\beta$ III tubulin. ${ }^{* *} P<0.001,{ }^{* * *} P<0.0001 . n=5$. One-way ANOVA with Tukey's post hoc analysis (B, E, and G). CTR, control; SWT, shock wave therapy; HPF, high-powered field.

In conclusion, we show a crucial mechanism of neuroprotection and spinal cord repair via TLR3, revealing SWT as a potent future treatment option for SCI. SWT is currently in routine clinical use for other indications and could therefore efficiently be directly translated to the treatment of traumatic SCI.

\section{Methods}

\section{Animals}

In this study, male 12-week-old C57BL/ 6 mice (Charles River) and TLR3-knockout mice (provided by Shizuo Akira, Laboratory of Host Defense, Research Institute for Microbial Disease, Osaka University, Osaka, Japan) weighing 18-25 g were used. They were randomly assigned to 1 of the following 4 groups: CTR 72 hours, CTR 10 weeks, SWT 72 hours, and SWT 10 weeks. The sample size of each 
A

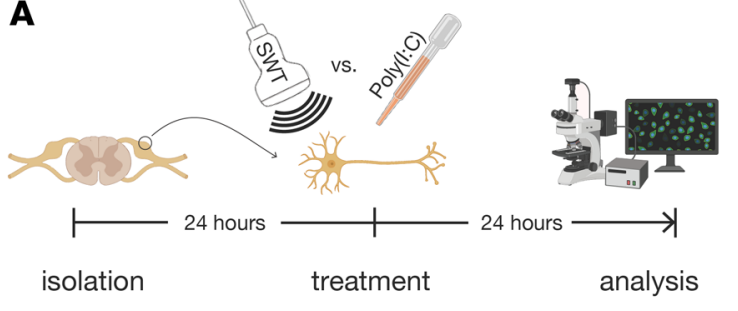

B

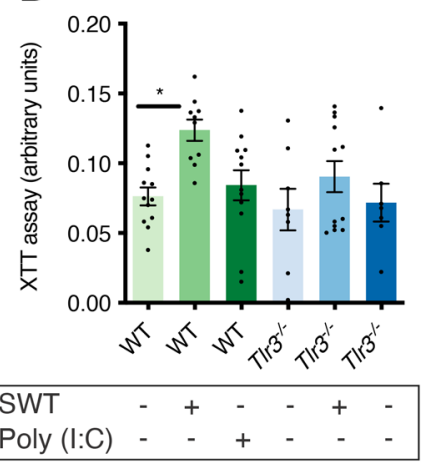

C

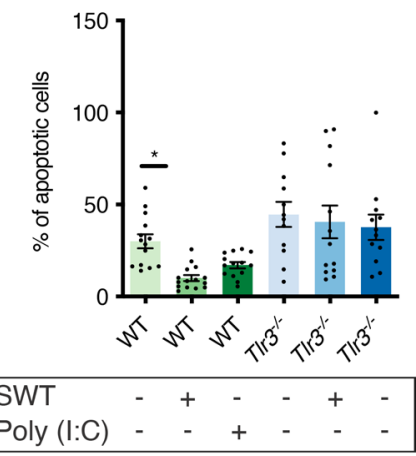

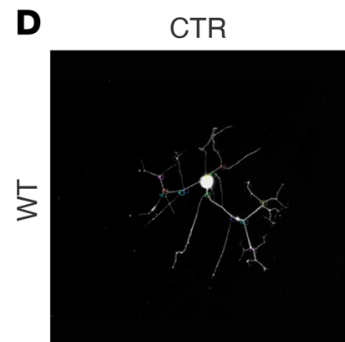

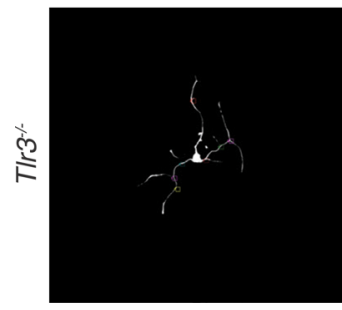

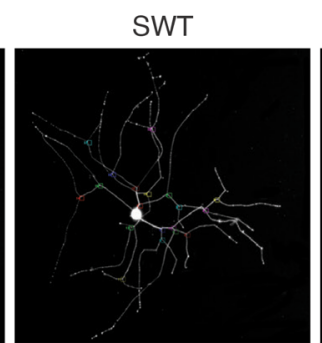

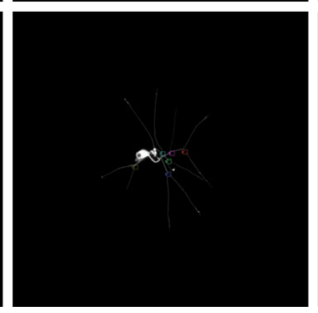

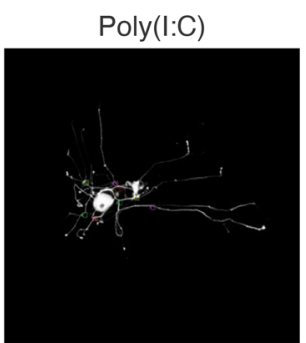

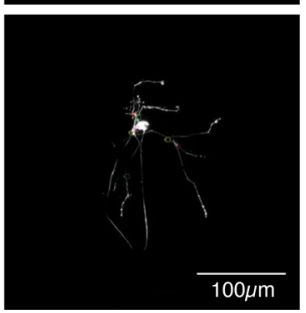

$\mathbf{E}$

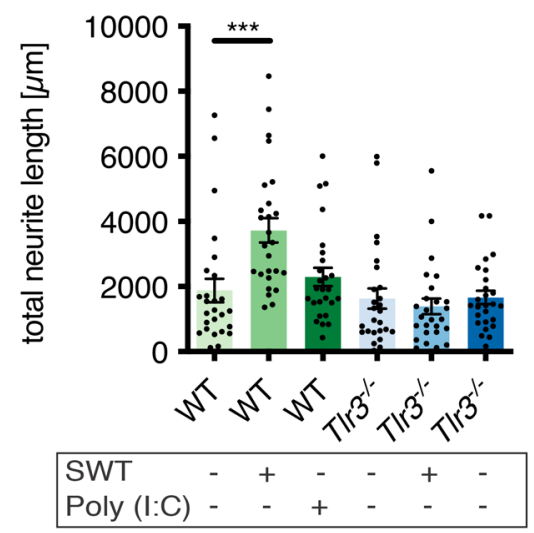

Figure 6. SWT reduces apoptosis and enhances neuronal sprouting. (A) Murine DRGs were isolated from WT and TIr $3^{-/-}$mice and assessed with in vitro assays for characterization of neuronal sprouting, metabolic activity, and apoptosis after SWT or poly(I:C) treatment. (B) XTT assay revealed improved viability of DRGs after SWT in WT but not T/r $3^{-1-}$ cells. Again, poly(I:C) had no effect on DRG cultures analyzed for viability. ${ }^{*} P<0.05 . n=$ 7-12. (C) DRGs were subjected to FCS starvation. SWT resulted in a significant reduction of apoptotic neurons, but only in WT cells. ${ }^{*} P<0.05 . n=$ 12-15. (D) Representative images of neuronal sprouting after SWT. Scale bar: $100 \mu \mathrm{m}$. (E) SWT stimulated neuronal sprouting in DRGs derived from WT mice. Sprouting was not stimulated in DRGs derived from $T / r 3^{-/-}$mice. Poly(I:C) stimulation showed no effect on neuronal sprouting in either WT or TIr $3^{-1-}$ cells. ${ }^{* * *} P<0.001 . n=26$. One-way ANOVA with Tukey's post hoc analysis (B, C, and E). CTR, control; SWT, shock wave therapy; XTT, (sodium 3'-[1-(phenylaminocarbonyl)-3,4-tetrazolium]-bis (4-methoxy6-nitro) benzene sulfonic acid hydrate).

group at each time point was at least 4. During the experiment, animals were housed under standardized conditions with water and commercial diet (ssniff, V1124-300) available ad libitum and a 12-hour light/dark cycle. Water and commercial mouse diet were available ad libitum.

\section{Murine clip contusion model}

For induction of SCI, a clip contusion model was used as described previously (31). Briefly, animals were anesthetized via intraperitoneal injection of $10 \%$ ketamine, $20 \%$ xylazine, and $70 \% \mathrm{NaCl}(0.01$ $\mu 1 / \mathrm{kg}$ body weight). After shaving and positioning, skin was incised along the dorsal midline, and paravertebral muscles between thoracic levels 7 and 10 were dissected. Under visual guidance, using a microscope (Carl Zeiss), T9 laminae were removed to reveal the spinal cord, leaving the dura intact. In order to cause a compression injury, a vascular clip (Aesculap) was applied to the exposed spinal cord for 60 seconds. Subsequently, muscles, fascia, and skin were adapted. After surgery, animals were placed under a heat lamp and supplied with oxygen until an alert state was reestablished. Manual bladder expression was performed twice daily for 1 week as described previously (7). Tissue samples were harvested either 72 hours or 10 weeks after SWT. 

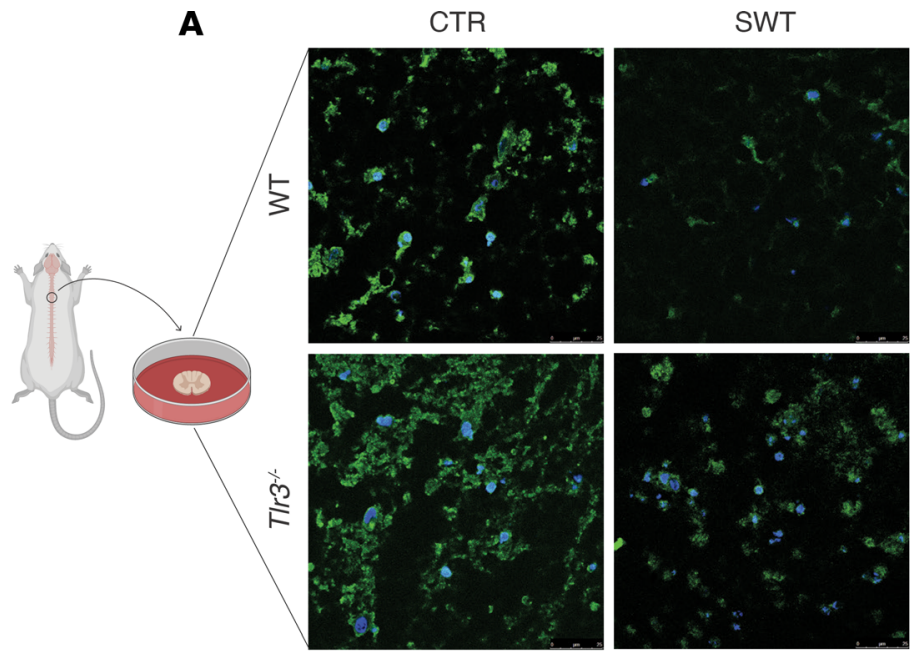

FJB DAPI
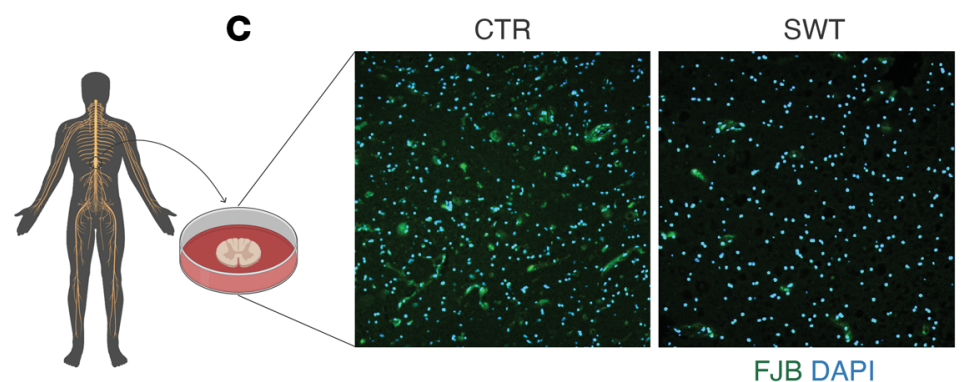

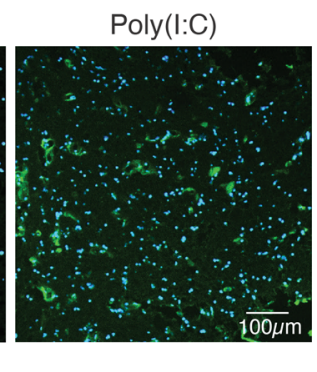

B
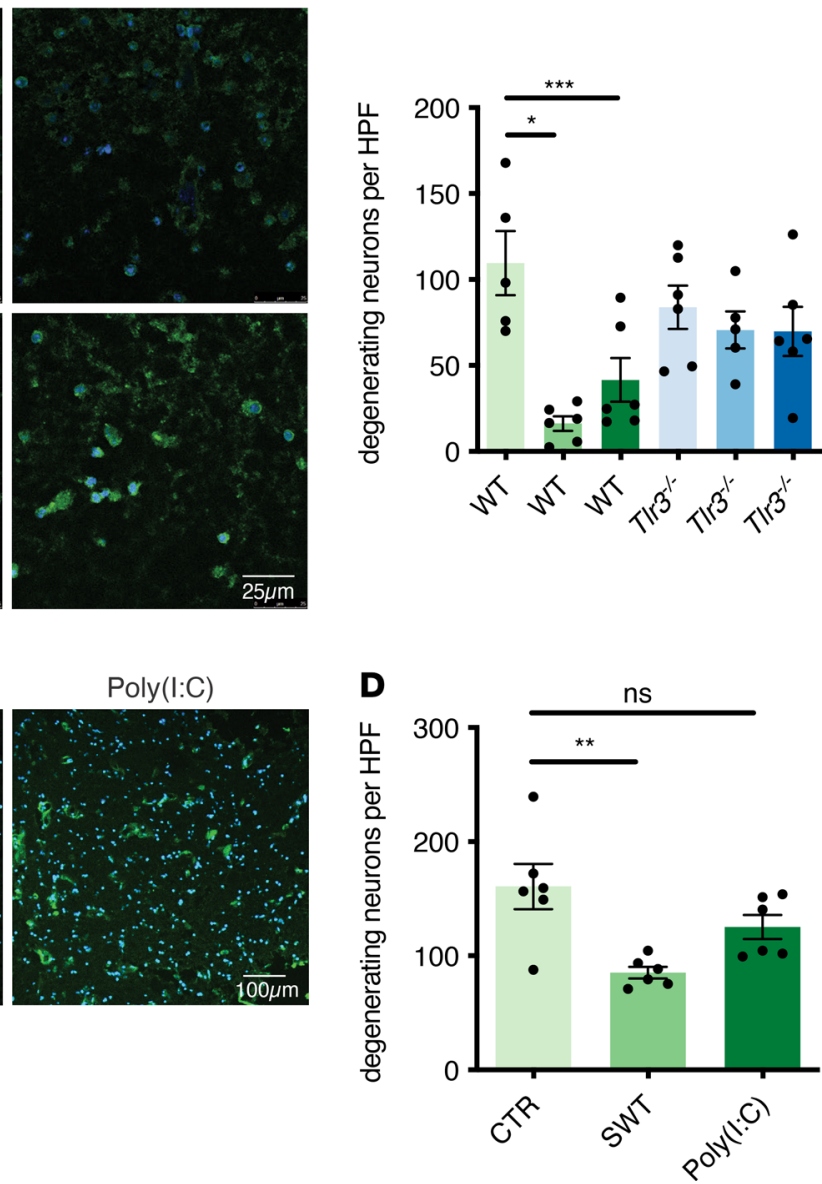

Figure 7. Reduction of neuronal degeneration in murine and human spinal slice cultures. (A) We performed a murine spinal slice culture to investigate the effect of SWT or poly(I:C) in tissue cultivating spinal slices from both WT and T/r $3^{-1-}$ mice. Scale bar: $25 \mu \mathrm{m}$. (B) Both SWT and poly(I:C) reduced the numbers of degenerating neurons significantly in WT but not $T / r 3^{-/-}$mice. ${ }^{*} P<0.05,{ }^{* *} P<0.001 . n=5-6$ animals. (C) To extend our investigation to a human model, we examined the effect of SWT in human spinal slice cultures obtained from the upper cervical segments of the spinal cord. Scale bar: $100 \mu \mathrm{m}$. (D) Consistent with our previous results, both SWT and poly $(I: C)$ reduced the number of degenerating neurons. ${ }^{* *} P<0.01 . n=6$ slices. One-way ANOVA with Tukey's post hoc analysis (B and D). CTR, control; SWT, shock wave therapy; FJB, Fluoro-Jade B.

\section{SWT}

After contusion injury, animals were left untreated for 14 days to obtain a state of chronic SCI. After 14 days, animals were randomly assigned to therapy or control groups. Animals in the treatment group underwent SWT. For this purpose, animals were anesthetized as described above. Ultrasound gel was used for coupling of the shock wave applicator. Subsequently, we applied 500 impulses at an energy flux density of $0.1 \mathrm{~mJ} / \mathrm{mm}^{2}$ and a frequency of $5 \mathrm{~Hz}$ using the Orthogold device with applicator CG050-P (Tissue Regeneration Technologies LLC) as described previously (1). The parameters of SWT were chosen due to previous experiments (1).

All in vitro SWTs were performed under standardized conditions as described previously (32) (300 impulses with an energy flux density of $0.08 \mathrm{~mJ} / \mathrm{mm}^{2}$ at a frequency of $3 \mathrm{~Hz}$ ).

\section{Assessment of motor function}

Behavioral tests were performed only after ethical approval. If animals were not willing or able to perform the behavioral test after 3 attempts, they were excluded from the analysis. The following methods were used for the assessment.

Gait analysis. Gait analysis was performed 10 weeks after treatment, as described previously (33). Briefly, a DigiGait Analysis System (Mouse Specifics) was used. Mice were placed upon a motorized transparent treadmill at the speed of $25 \mathrm{~cm} / \mathrm{s}$ from underneath. Processing of the data was performed 
using the specific DigiGait Software 9.0 (Mouse Specifics). As motor function of hind limbs was severely compromised after contusion injury, automated measurement of gait was not possible. However, we performed blinded semiquantitative analysis of gait videos as described previously (34).

Open-field activity. Locomotor activity was tested using the open-field analysis 10 weeks after treatment as described previously (35). Briefly, animals were placed at the center of an open field $(40.5 \times 40.5 \times 36.5$ $\mathrm{cm}$ ) and analyzed for 15 minutes in a dark room isolated from noise and light. A Flex Field Activity System (San Diego Instruments) was used to quantify horizontal and vertical locomotor activity and rearing.

\section{MRI imaging}

High resolution 3D MRI was performed on a 3 Tesla whole-body scanner (Magnetom Skyra) with a 45 $\mathrm{mT} / \mathrm{m}$ gradient system. Mice were positioned prone within a combination of 2 small loop coils with an inner diameter of $3 \mathrm{~cm}$ (standard finger coils). Sagittal proton density-weighted images with fat suppression were acquired using a driven equilibrium 3D SPACE sequence with the following parameters: TR, $1000 \mathrm{~ms}$; TE, $49 \mathrm{~ms}$; echo train length, 18; field of view (FOV), $68 \times 99 \mathrm{~mm}$; acquisition matrix, $175 \times 256$; number of images, 40 (no gap); voxel size, $0.387 \times 0.387 \times 0.4 \mathrm{~mm}$; number of averages, 2 ; acquisition time, 9.11 minutes. In addition T2-weighted images, also in sagittal orientation and with fat saturation, were acquired using a fast spin-echo sequence with TR, $5080 \mathrm{~ms}$; TE, $70 \mathrm{~ms}$; echo train length, 14; FOV, $70 \times 70$ $\mathrm{mm}$; acquisition matrix, $320 \times 240$; number of images, 13 ; slice thickness, $0.7 \mathrm{~mm}$; spacing between slices, $0.77 \mathrm{~mm}$; voxel size, $0.219 \times 0.219 \times 7 \mathrm{~mm}$; number of averages, 4 ; acquisition time, 6.00 minutes. During the acquisition, mice were sedated via intraperitoneal injection of $10 \%$ ketamine, $20 \%$ xylazine, and $70 \%$ $\mathrm{NaCl}$, with a dosage of 0.01 proton density-weighted $\mu \mathrm{L} / \mathrm{kg}$ bodyweight. ROI segmentation was used to generate both spinal scar and bladder volumes as described previously (36). Volumes were quantified thereafter using Osirix Software for Mac (version 9.5).

\section{Immunohistochemistry}

Immunohistochemistry of samples was performed as described previously (1). Briefly, tissue samples were fixed in $4 \%$ PFA, embedded in paraffin, and cut into $5 \mu \mathrm{m}$ sections.

After heat-mediated antigen retrieval (Vector antigen unmasking solution; Vector Laboratories), samples were blocked with $1 \%$ BSA for 30 minutes at room temperature. Subsequently, slides were incubated with polyclonal rabbit anti-TLR3 (MilliporeSigma) or monoclonal mouse anti-nestin (Abcam) antibodies overnight at $4^{\circ} \mathrm{C}$. For signal detection, secondary antibodies (Alexa Fluor 568/488; Life Technologies) were incubated for 1 hour at room temperature. Nuclear staining was performed using DAPI (Life Technologies) according to the manufacturer's protocols. For Fluoro-Jade B staining, a commercially available staining kit (AG31030MG, Merck Millipore) was used as recommended by the manufacturer. H\&E staining was performed as described previously for histological quantification of spinal cord damage (37). Analysis of 5 slides per animal was performed in a blinded fashion. For each slide, 5 randomly chosen fields (high-power fields) were photographed. An Olympus IX70 microscope (Olympus) or a broadband Confocal Leica TCS SP5 microscope (lens: HCX PL APO CS $63 \times 1.2$ [glycerine]) (Leica) was used. Quantification was performed using ImageJ (NIH). Images were analyzed independently by 2 blinded researchers for anti-nestin-immunopositive cells. Images without concordance were excluded. Severity scores in transversal histopathological sections were quantified as follows: 0 , no lesion; 1 , mild lesion (focal disruption of myelin sheaths and axons); 2 , moderate lesion; 3 , severe lesion (diffuse disruption of myelin sheath and axons), as described previously (38).

\section{Primary neuron culture}

DRG from 8- to 10-week-old mice were isolated as described previously (39). Briefly, after removal of connective tissue ganglia were incubated in liberase blendzyme 1 ( 9 mg/100 mL DMEM, Roche) for 60 minutes. Trypsin-EDTA was added for 15 minutes after a rinse in PBS (Life Technologies). Subsequently, DRGs were washed with TNB 100 medium (Biochrom) supplemented with L-glutamine (Life Technologies), penicillin G sodium, streptomycin-sulfate (Life Technologies), and protein-lipid complex (Biochrom).

Dissociation of the ganglia was performed using a fire-polished Pasteur pipette. To minimize contamination with nonneural cells, centrifugation at low speed (10 minutes, $47 \mathrm{~g}$ ) through a $3.5 \%$ BSA gradient (MilliporeSigma) was performed. Thereafter, neurons were plated at a density of 50-80 per glass coverslip coated with poly-L-lysine/laminin (MilliporeSigma) and cultivated in TNB medium at $37^{\circ} \mathrm{C}$ in $5 \% \mathrm{CO}_{2}$. Cells were treated with SWT or poly(I:C) (MilliporeSigma) under standardized conditions, as described 
previously (22). Functional assays, including neuronal sprouting assay, XTT assay, and TUNEL assay, were performed 24 hours after treatment as described elsewhere (40-42).

\section{Neuroblastoma cell culture}

A neuroblastoma cell line was used to investigate the effect of SWT on neuronal function in vitro. Therefore, SH-SY5Y cells (ATCC) were cultivated in DMEM with $4.5 \mathrm{~g} / \mathrm{L}$ Glucose (PAN Biotech) with 10\% (vol/vol) FCS and 1\% penicillin-streptomycin solution (both from MilliporeSigma). Cells were harvested for gene expression analysis at 2, 4, 6, 12, and 24 hours upon SWT.

\section{Spinal slice culture}

Spinal slice culture was performed as described previously (1). Briefly, spinal cords from 12-week-old C57BL/6 WT mice (Charles River) and from $\operatorname{Tlr}^{-1-}$ mice (provided by Shizuo Akira) were harvested and dissected into $1 \mathrm{~cm}$ slices. Spinal cord slices (including human samples) were cultured in DMEM high glucose (Life Technologies) containing 10\% (vol/vol) FCS and 1\% penicillin-streptomycin solution (both from MilliporeSigma) and $5 \mathrm{ng} / \mathrm{mL}$ mouse nerve growth factor (Alomone Laboratories). The SWT group received SWT (300 impulses with an energy flux density of $0.08 \mathrm{~mJ} / \mathrm{mm}^{2}$ at a frequency of $3 \mathrm{~Hz}$ ) as described previously (1). After 24 hours, samples were embedded in Tissue-Tek (Sakura), and microsection for immunofluorescence was performed.

\section{Human samples}

This study used a nonembalmed cadaver with a postmortem time of 2 hours as described previously (1). The human cadaver (male) was donated to the Division of Clinical and Functional Anatomy, Department for Anatomy, Histology, and Embryology of the Medical University of Innsbruck. Sampling was performed from the upper cervical segments of the spinal cord.

\section{Zebrafish model}

The zebrafish model was performed as described previously (13) with some minor modifications.

Lesions. 96 hpf larvae were anesthetized in E3 medium containing 0.02\% tricaine (MS222; MilliporeSigma). Larva were transferred to a plastic Petri dish, and following aspiration of excess water, the tip of a 26 gauge syringe needle was used to inflict a lesion in the dorsal trunk at the level of the anal pore. After the lesion was performed, larvae were washed briefly in E3 medium to remove tricaine. For recovery 15-20 larva were pooled per 6 well pate and incubated over night at $28^{\circ} \mathrm{C}$ in $4 \mathrm{ml} \mathrm{E} 3$ medium containing $0.1 \%$ DMSO and different compounds. Final concentration of compounds was $5 \mathrm{mg} / \mathrm{mL}$ for TLR3 inhibitor (TLR3/dsRNA complex inhibitor, Merck) and $100 \mu \mathrm{g} / \mathrm{mL}$ for poly(I:C) (MilliporeSigma). The next day $120 \mathrm{hpf}$ larva (>93\% survival) were inspected by light microscopy to detect and exclude larva showing extensive damage to the notochord $(\sim 10 \%)$. Remaining larva were either further analyzed for mobility, or they were fixed overnight in 4\% PFA. After fixation larva were briefly washed in PBT (PBS containing 0.1\% Tween-20) and stepwise dehydration by successive incubation in 25\% MeOH-PBT, 50\% MeOH-PBT, 75\% MeOH-PBT, and 100\% MeOH for 5 minutes each. Larva were stored at $-20^{\circ} \mathrm{C}$ until needed.

Immunolabeling. Whole-mount immunolabeling of $120 \mathrm{hpf}$ larva for acetylated Tubulin/GFAP (T7451, MilliporeSigma) and A-11030, Thermo Fisher, both 1:1000) was performed as described previously (13).

Confocal image analyses. A Zeiss LSM Exciter 5 was used to image immunostained sites, using a $40 \times / 0.80 \mathrm{~W}$ ACHROPLAN objective (Carl Zeiss). Lesion sites were documented as confocal image stacks using standardized settings. In order to evaluate neuronal volume, acetylated tubulin signals at the lesion site were quantified using the ImageJ plugin "color pixel counter." In the first step, signal intensities were normalized to motoaxon signals (spinal cord exit points) to compensate for experiment to experiment variation. Next, a $313 \times 215$ pixel ROI containing the lesion site and spanning the entire spinal cord was selected to determine number of voxels with signal intensities above $8 \%$ of maximum intensity.

Mobility assay. Provoked larva mobility was stimulated at the tail fin with a hair attached to a preparation needle. Two larva at a time were transferred to a Petri dish with shallow water to restrict movement to the $x-y$ plane. Movement was documented with $10 \mathrm{~Hz}$ over an interval of 8 seconds using a Ipevo ZIGGI HD+ document camera. Fish were tracked by hand using the "Manual Tracking" ImageJ plugin (NIH). The end of the movement was defined when the fish remained in the same position for at least $300 \mathrm{~ms}$. 
Table 1. Antibodies

\begin{tabular}{cc}
\hline Antibody & Source; catalog no. \\
TLR3 & abcam; ab62566 \\
Nestin & abcam; ab11306 \\
GAPDH (6C5) & Invitrogen; AM4300 \\
\hline
\end{tabular}

\section{NPC isolation}

NPCs were isolated from spinal cords from 12-week-old C57BL/6 WT mice (Charles River) and from $\mathrm{Tlr}^{-1-}$ mice (provided by Shizuo Akira) as described previously (43). Briefly, after euthanization spinal cords were minced and incubated in trypsin for 30 minutes at $37^{\circ} \mathrm{C}$. Cells were filtered $(400 \mathrm{~nm}$ filter, Corning) and centrifuged in sucrose for 30 minutes at $750 \mathrm{~g}$, and the myelin layer was removed upon seeding in gelatin-coated flasks. NPCs were cultured as described previously (44). The presence of nestin was verified using immunofluorescence staining (monoclonal mouse anti-nestin, abcam).

\section{Differentiation assay}

For assessment of differentiation properties, NPCs isolated from WT or TLR3 $3^{-1-}$ mice were treated with either SWT as described above or poly(I:C), seeded in laminin-precoated 24 wells, and cultured in differentiation medium (DMEM/F12 medium, Life Technologies) containing 5\% (vol/vol) fetal bovine serum, 1\% (vol/vol) N2 supplement (Life Technologies) and penicillin-streptomycin solution for 5 days as described previously (44). Subsequently, cells were labeled with monoclonal mouse anti- $\beta$ III tubulin antibody (R\&D Systems) for identification of neurons as described previously (45).

\section{Western blotting}

Western blotting was performed as described previously (22). Primary antibodies used for analysis are described in Table 1.

\section{RT-PCR}

RT-PCR was performed as described previously (22). Primers used in the experiments are described in Table 2.

\section{Proliferation}

A chemiluminescent BrdU assay kit (abcam) was used as recommended by the manufacturer. Briefly, 4000 cells were seeded in gelatin-precoated 96 well plates. Eleven hours after therapy, cells were labeled with BrdU for another 11 hours and analyzed subsequently.

\section{Gene expression profiling}

Total RNA was isolated from 4 samples of SH-SY5Y cells and was submitted to transcriptome analysis for the purpose of gene-expression profiling. The 2100 Bioanalyzer and RNA 6000 Nano LabChip

Table 2. Primers

\begin{tabular}{|c|c|}
\hline \multirow{2}{*}{ Human TLR3 } & 5'-AGGAAAGGCTAGCAGTCATCC-3' \\
\hline & 5'-TAACAGTGCACTTGGTGGTG-3' \\
\hline \multirow{2}{*}{ Human TRIF } & 5'-CGGGCAACTCCGCAGAAG-3' \\
\hline & 5'-СATTCACTCTTCCAGGTTCTCC-3' \\
\hline \multirow{2}{*}{ Human IL-6 } & 5'-ACCCACTCACCTCTTCACAAC-3' \\
\hline & 5'-AGTCСCTCTTTGCTGCTTTC-3' \\
\hline \multirow{2}{*}{ Human IL-6-receptor } & 5'-GTAGCCGAGGAGGAAGCATG-3' \\
\hline & 5'-TCTCCTCGCAGACTGGTCAG-3' \\
\hline \multirow{2}{*}{ Human GAPDH } & 5'-GGTGGTCTCCTCTGACTTCAACA-3' \\
\hline & 5'-GTGGTCGTTGAGGGCAATG-3' \\
\hline
\end{tabular}


kits (Agilent Technologies) were used to check for RNA integrity (all RINs $>9$ ). The TruSeq Stranded mRNA HT technology was used for library preparation according to manufacturer's protocols and Illumina NextSeq $5001 \times 75$ bp single-end sequencing was performed at the IMGM laboratories. Adapters from single-end reads (FASTQ files) were removed, and reads were cropped at a length of $73 \mathrm{bp}$ using Trimmomatic (0.36) (46). Sequencing quality was checked using FastQC (0.11.5). Reads were mapped using STAR aligner (2.5.3a) (47) on human genome version hg38 (UCSC) with RefGene annotation and indexed with $100 \mathrm{bp}$ splice junction overhang. HTSeq (48) served to quantify gene raw counts. DESeq2 (49) within the R environment (3.4.1) was used to identify differentially expressed genes using negative binomial distribution filtered for genes with normalized mean counts $>20$ across the compared samples. Overrepresentation analysis for gene ontology and pathways on differentially expressed genes was performed using DAVID (6.8) (50) and ConsensusPathDB (51). Heatmaps were generated using Genesis version 1.8 .1 on gene-wise $Z$ score transformed data (52). Raw data were deposited in a public repository (GEO accession GSE149446).

\section{Statistics}

All results are expressed as mean \pm SEM. Statistical comparisons between 2 groups were performed by 2 -tailed Student's $t$ or Mann-Whitney test as appropriate. Multiple groups were analyzed by 1-way ANOVA with Tukey's post hoc analysis to determine statistical significance. Probability values of $P<0.05$ were considered statistically significant.

\section{Study approval}

Experiments were approved by the Austria animal care and use committee (BMWFW 66.011/0108-WF/V/3b/2016) and conformed to the Guide for the Care and Use of Laboratory Animals (National Academies Press, 2011). Informed consent was given for use of the cadaver for scientific and educational purposes.

\section{Author contributions}

CGT, FN, JH, NS, DM, and IT conceived the study and designed the research. CGT, FN, MG, LP, DL, JH, AA, RI, BR, CK, HH, RH, SV, and HF acquired the data. CGT, FN, NS, DM, NB, MG, and $\mathrm{JH}$ analyzed and interpreted the data. CGT and $\mathrm{JH}$ provided statistical analysis. $\mathrm{JH}$ and MG obtained funding and supervising the work. CGT, JH, IT, and FN drafted the manuscript. DH, HF, NB, NS, IT, $\mathrm{DM}$, and MG provided critical revision of the manuscript for important intellectual content.

\section{Acknowledgments}

This work was supported by an AUVA unrestricted research grant to JH and CGT and the Austrian Science Fund (F4414 to NS).

Address correspondence to: Johannes Holfeld, Department of Cardiac Surgery, Medical University of Innsbruck, Anichstr.35, 6020 Innsbruck, Austria. Phone: 43.512.504.80800; Email: Johannes.holfeld@i-med.ac.at.

1. Lobenwein D, et al. Shock wave treatment protects from neuronal degeneration via a Toll-like receptor 3 dependent mechanism: implications of a first-ever causal treatment for ischemic spinal cord injury. J Am Heart Assoc. 2015;4(10):e002440.

2. Baaj AA, et al. Health care burden of cervical spine fractures in the United States: analysis of a nationwide database over a 10-year period. J Neurosurg Spine. 2010;13(1):61-66

3. National Spinal Cord InjuryStatistical Center. Spinal cord Injury facts and figures at a glance. J Spinal Cord Med 2014;37(3):355-356

4. Orr MB, Gensel JC. Spinal cord injury scarring and inflammation: therapies targeting glial and inflammatory responses. Neurotherapeutics. 2018;15(3):541-553.

5. Cavassani KA, et al. TLR3 is an endogenous sensor of tissue necrosis during acute inflammatory events. J Exp Med. 2008;205(11):2609-2621.

6. Bernard JJ, et al. Ultraviolet radiation damages self noncoding RNA and is detected by TLR3. Nat Med. 2012;18(8):1286-1290.

7. Nelson AM, et al. dsRNA released by tissue damage activates TLR3 to drive skin regeneration. Cell Stem Cell. 2015;17(2):139151.

8. Ottomann C, et al. Prospective randomized phase II Trial of accelerated reepithelialization of superficial second-degree burn wounds using extracorporeal shock wave therapy. Ann Surg. 2012;255(1):23-29.

9. Wang CJ, Yang KD, Wang FS, Hsu CC, Chen HH. Shock wave treatment shows dose-dependent enhancement of bone mass 
and bone strength after fracture of the femur. Bone. 2004;34(1):225-230.

10. Gollmann-Tepeköylü C, et al. Shock wave therapy improves cardiac function in a model of chronic ischemic heart failure: evidence for a mechanism involving VEGF signaling and the extracellular matrix. J Am Heart Assoc. 2018;7(20):e010025.

11. Taweel WA, Seyam R. Neurogenic bladder in spinal cord injury patients. Res Rep Urol. 2015;7:85-99.

12. Qian J, Herrera JJ, Narayana PA. Neuronal and axonal degeneration in experimental spinal cord injury: in vivo proton magnetic resonance spectroscopy and histology. J Neurotrauma. 2010;27(3):599-610.

13. Wehner D, et al. Wnt signaling controls pro-regenerative Collagen XII in functional spinal cord regeneration in zebrafish. Nat Commun. 2017;8(1):126.

14. Ghosh S, Hui SP. Axonal regeneration in zebrafish spinal cord. Regeneration (Oxf). 2018;5(1):43-60.

15. Chapela D, et al. A zebrafish drug screening platform boosts the discovery of novel therapeutics for spinal cord injury in mammals. Sci Rep. 2019;9(1):10475.

16. Oosterhof N, et al. Identification of a conserved and acute neurodegeneration-specific microglial transcriptome in the zebrafish. Glia. 2017;65(1):138-149.

17. Storer MA, Gallagher D, Fatt MP, Simonetta JV, Kaplan DR, Miller FD. Interleukin-6 regulates adult neural stem cell numbers during normal and abnormal post-natal development. Stem Cell Reports. 2018;10(5):1464-1480.

18. Takeuchi O, Akira S. Pattern recognition receptors and inflammation. Cell. 2010;140(6):805-820.

19. Bradbury EJ, McMahon SB. Spinal cord repair strategies: why do they work? Nat Rev Neurosci. 2006;7(8):644-653

20. Sofroniew MV. Dissecting spinal cord regeneration. Nature. 2018;557(7705):343-350.

21. Bsibsi M, Persoon-Deen C, Verwer RW, Meeuwsen S, Ravid R, Van Noort JM. Toll-like receptor 3 on adult human astrocytes triggers production of neuroprotective mediators. Glia. 2006;53(7):688-695.

22. Holfeld J, et al. Toll-like receptor 3 signalling mediates angiogenic response upon shock wave treatment of ischaemic muscle. Cardiovasc Res. 2016;109(2):331-343.

23. Tepeköylü C, et al. Shockwaves prevent from heart failure after acute myocardial ischaemia via RNA/protein complexes. J Cell Mol Med. 2017;21(4):791-801.

24. Bsibsi M, Ravid R, Gveric D, van Noort JM. Broad expression of Toll-like receptors in the human central nervous system. J Neuropathol Exp Neurol. 2002;61(11):1013-1021.

25. Kono H, Rock KL. How dying cells alert the immune system to danger. Nat Rev Immunol. 2008;8(4):279-289.

26. Gollmann-Tepeköylü C, et al. miR-19a-3p containing exosomes improve function of ischaemic myocardium upon shock wave therapy. Cardiovasc Res. 2020;116(6):1226-1236.

27. Vicencio JM, et al. Plasma exosomes protect the myocardium from ischemia-reperfusion injury. J Am Coll Cardiol. 2015;65(15):1525-1536.

28. Bell MT, et al. Toll-like receptor 4-dependent microglial activation mediates spinal cord ischemia-reperfusion injury. Circulation. 2013;128(11 Suppl 1):S152-S156.

29. Yang P, Wen H, Ou S, Cui J, Fan D. IL-6 promotes regeneration and functional recovery after cortical spinal tract injury by reactivating intrinsic growth program of neurons and enhancing synapse formation. Exp Neurol. 2012;236(1):19-27.

30. Akira S, Uematsu S, Takeuchi O. Pathogen recognition and innate immunity. Cell. 2006;124(4):783-801.

31. Marques SA, Garcez VF, Del Bel EA, Martinez AM. A simple, inexpensive and easily reproducible model of spinal cord injury in mice: morphological and functional assessment. J Neurosci Methods. 2009;177(1):183-193.

32. Holfeld J, et al. Low energy shock wave therapy induces angiogenesis in acute hind-limb ischemia via VEGF receptor 2 phosphorylation. PLoS One. 2014;9(8):e103982.

33. Sturm E, Fellner L, Krismer F, Poewe W, Wenning GK, Stefanova N. Neuroprotection by epigenetic modulation in a transgenic model of multiple system atrophy. Neurotherapeutics. 2016;13(4):871-879.

34. Guertin PA. Semiquantitative assessment of hindlimb movement recovery without intervention in adult paraplegic mice. Spinal Cord. 2005;43(3):162-166.

35. Stefanova N, Kaufmann WA, Humpel C, Poewe W, Wenning GK. Systemic proteasome inhibition triggers neurodegeneration in a transgenic mouse model expressing human $\alpha$-synuclein under oligodendrocyte promoter: implications for multiple system atrophy. Acta Neuropathol. 2012;124(1):51-65.

36. Fandel TM, et al. Transplanted human stem cell-derived interneuron precursors mitigate mouse bladder dysfunction and central neuropathic pain after spinal cord injury. Cell Stem Cell. 2016;19(4):544-557.

37. Oller J, et al. Nitric oxide mediates aortic disease in mice deficient in the metalloprotease Adamts1 and in a mouse model of Marfan syndrome. Nat Med. 2017;23(2):200-212.

38. Ji J, Yan X, Li Z, Lai Z, Liu J. Therapeutic effects of intrathecal versus intravenous monosialoganglioside against bupivacaine-induced spinal neurotoxicity in rats. Biomed Pharmacother. 2015;69:311-316.

39. Marvaldi L, et al. Enhanced axon outgrowth and improved long-distance axon regeneration in sprouty 2 deficient mice. Dev Neurobiol. 2015;75(3):217-231

40. Theurl M, et al. The neuropeptide catestatin acts as a novel angiogenic cytokine via a basic fibroblast growth factor-dependent mechanism. Circ Res. 2010;107(11):1326-1335.

41. Suarez V, et al. The axotomy-induced neuropeptides galanin and pituitary adenylate cyclase-activating peptide promote axonal sprouting of primary afferent and cranial motor neurones. Eur J Neurosci. 2006;24(6):1555-1564.

42. Aras MA, Hartnett KA, Aizenman E. Assessment of cell viability in primary neuronal cultures. Curr Protoc Neurosci. 2008; Chapter 7:Unit 7.18.

43. Hugnot JP. Isolate and culture neural stem cells from the mouse adult spinal cord. Methods Mol Biol. 2013;1059:53-63.

44. Theus MH, Ricard J, Liebl DJ. Reproducible expansion and characterization of mouse neural stem/progenitor cells in adherent cultures derived from the adult subventricular zone. Curr Protoc Stem Cell Biol. 2012; Chapter 2:Unit 2D.8.

45. Rolls A, et al. Toll-like receptors modulate adult hippocampal neurogenesis. Nat Cell Biol. 2007;9(9):1081-1088.

46. Bolger AM, Lohse M, Usadel B. Trimmomatic: a flexible trimmer for Illumina sequence data. Bioinformatics.

2014;30(15):2114-2120.

47. Dobin A, et al. STAR: ultrafast universal RNA-seq aligner. Bioinformatics. 2013;29(1):15-21. 
48. Anders S, Pyl PT, Huber W. HTSeq--a Python framework to work with high-throughput sequencing data. Bioinformatics. 2015;31(2):166-169

49. Love MI, Huber W, Anders S. Moderated estimation of fold change and dispersion for RNA-seq data with DESeq2. Genome Biol. 2014;15(12):550.

50. Huang da W, Sherman BT, Lempicki RA. Systematic and integrative analysis of large gene lists using DAVID bioinformatics resources. Nat Protoc. 2009;4(1):44-57.

51. Kamburov A, Pentchev K, Galicka H, Wierling C, Lehrach H, Herwig R. ConsensusPathDB: toward a more complete picture of cell biology. Nucleic Acids Res. 2011;39(Database issue):D712-D717.

52. Sturn A, Quackenbush J, Trajanoski Z. Genesis: cluster analysis of microarray data. Bioinformatics. 2002;18(1):207-208. 